\title{
Dissociable brain components for inhibitory control: A source separation analysis of cancelling and
} stopping actions.

\author{
Mario Hervault*1, Pier-Giorgio Zanone ${ }^{1}$, Jean-Christophe Buisson ${ }^{2}$, Raoul Huys ${ }^{1}$ \\ ${ }^{1}$ Brain and Cognition Research Center - UMR 5549 CNRS - University Toulouse III Paul Sabatier \\ ${ }^{2}$ Toulouse Research Institute in Computer Science - CNRS UMR5505 - University Toulouse III Paul Sabatier
}

\begin{abstract}
Previous work has drawn an inhibitory brain network by investigating the cancellation of prepareddiscrete actions, thus focusing on an isolated aspect of human behavior. Indeed, ongoing-continuous actions, which have been associated with distinct brain mechanisms for generation, are also crucial to be stopped. Still, it is unknown whether the same inhibitory network generalizes to various situations, including cancelling a prepared-discrete action and stopping an ongoing-continuous action. We used independent component analysis of EEG data to show that cancelling and stopping action rely on independent brain components for inhibition. Indeed, the brain component which best explained inhibition when cancelling action was not involved in stopping action, and reciprocally. The analysis of these components showed that a Delta/Theta power increase generically indexes inhibitory activity, while N2 and P3 event-related potentials do it in a specific way. Sources reconstruction further identified dissociations in the brain areas generating the two inhibitory components. The differences identified between the two source generators underlie some specific activities of the inhibitory network involved in cancelling a prepared-discrete action and stopping an ongoing-continuous action. Thus, increased activity was observed in precentral gyri and posterior parts of the cingulate cortex when action cancelling, while a higher activity was found in more frontal gyri and anterior parts of the cingulate cortex when action stopping. Overall, the present findings support the idea that inhibitory control is differentially implemented according to the type of action to revise.
\end{abstract}

Keywords Response inhibition, cognitive control, motor control, source separation

* Speaker and corresponding author:

Mario Hervault, CNRS CERCO UMR 5549, Pavillon Baudot CHU Purpan, 31052 Toulouse, FRANCE mario.hervault@cnrs.fr 


\title{
Dissociable brain components for inhibitory control: A source separation analysis of cancelling and stopping actions
}

\author{
Mario Hervault1, Pier-Giorgio Zanone1, Jean-Christophe Buisson², Raoul Huys' \\ 1 Centre de Recherche Cerveau et Cognition (CERCO) - CNRS UMR5549 - Université Toulouse 3 - France II \\ 2 Institut de Recherche en Informatique de Toulouse (IRIT) - CNRS UMR5505 - Université Toulouse 3 - France II \\ mario.hervault@cnrs.fr
}

The brain inhibitory network responsible for the crucial ability of action inhibition [1] has been investigated in the restricted context of discrete actions, using the stop-signal paradigm [2]

However, discrete and continuous actions are two fundamental classes of actions that engage distinct control processes and brain regions ${ }^{[3]}$, on which the inhibitory network acts through downstream effects [1].

In two experiments,14 participants had to cancel a prepared-discrete action or stop an ongoing-continuous action when facing an infrequent STOP signal.

Using a CIPI analysis ${ }^{[4]}$ (Fig. 1), we tested whether discrete-action cancelling and rhythmic-action stopping rely on the same brain component.

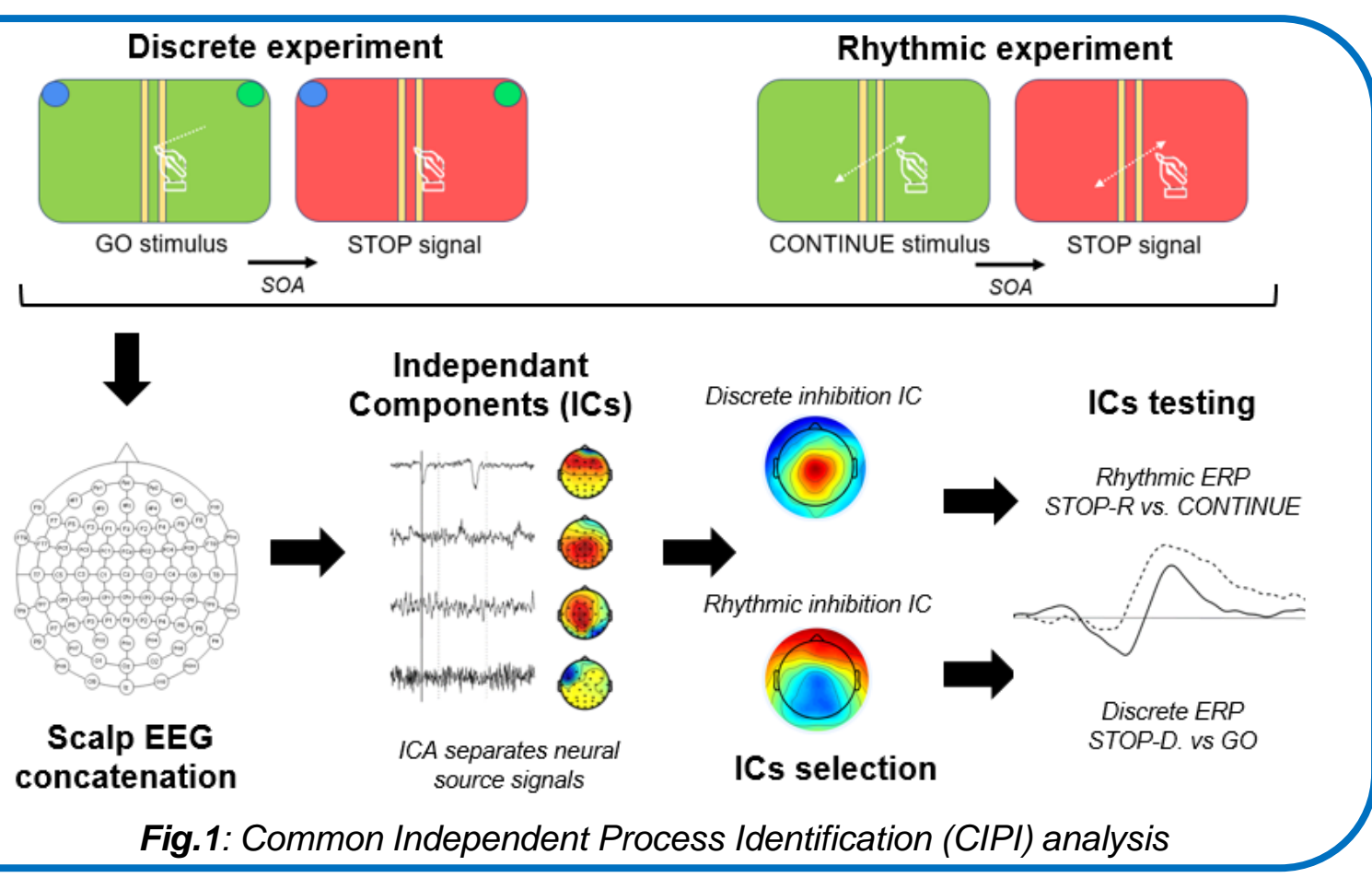

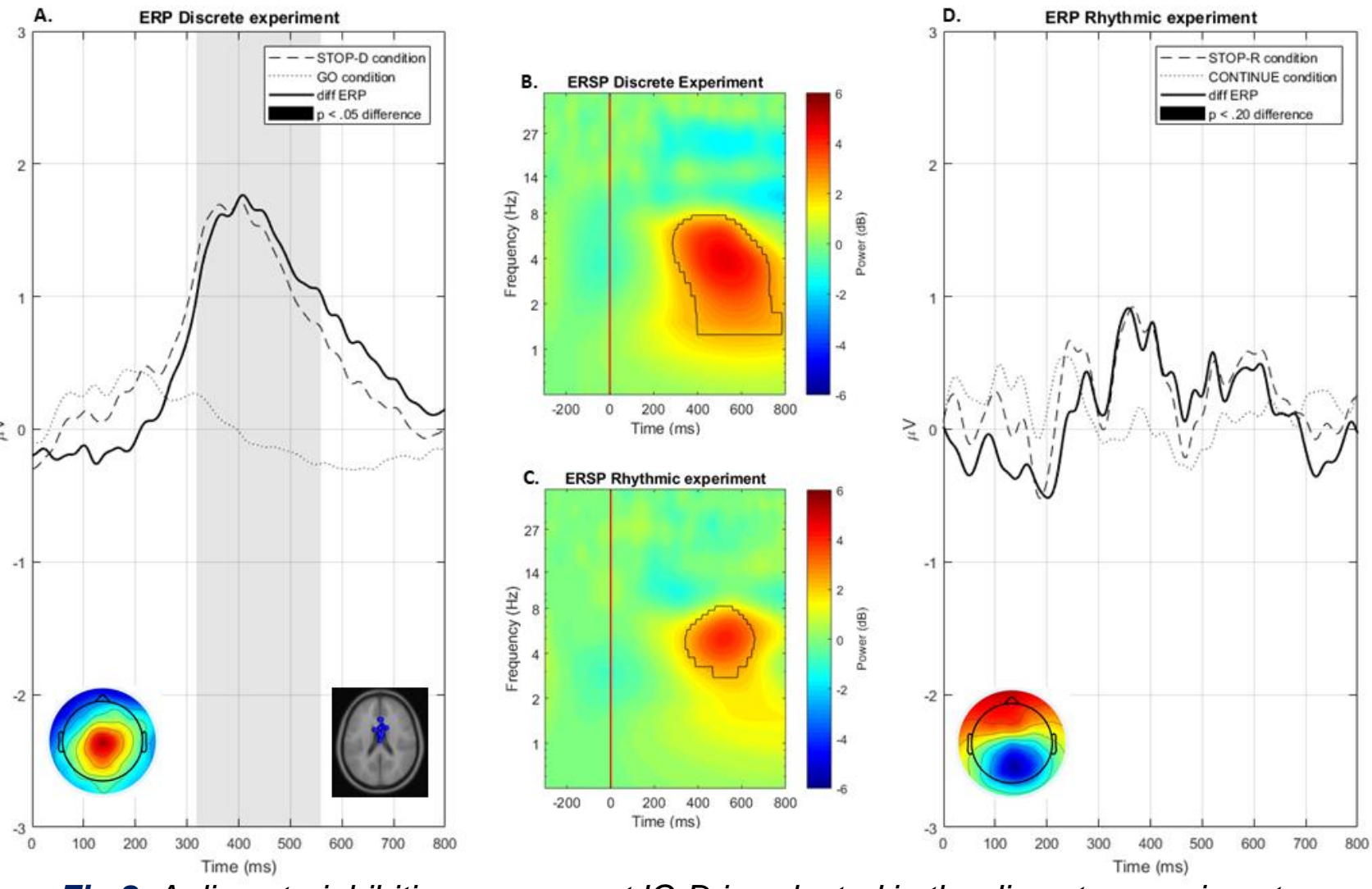

Fig.2: A discrete-inhibition component IC-D is selected in the discrete experiment and its activity is tested after rhythmic STOP signals (grand average plotted)

\section{Rhythmic-inhibition brain component analysis}

IC-R (Fig.3) is the single brain component that represents rhythmicaction inhibition, for each participant, by maximising rhythmic CONTINUE vs. STOP conditions difference.

$>$ In the rhythmic experiment : This IC-R showed a Delta/Theta power increase (panel B) and significant N2 and P3 ERP waves (panel $D$ ).

SSRT-R correlated to P3 peak amplitude $(r=-.65, p<.05)$.

$>$ In the discrete experiment : The same brain component (IC-R) did not significantly disentangle GO vs. STOP conditions (panel $A$ ).

\section{Discrete-inhibition brain component analysis}

IC-D (Fig.2) is the single brain component that best represents discrete-action inhibition, for each participant, by maximising discrete GO vs. STOP conditions ERP difference.

$>$ In the discrete experiment : This IC-D showed a Delta/Theta power increase (panel B) and a significant P3 ERP wave (panel A). Discrete stop-signal reaction time (SSRT-D) correlated to P3 onset latency $(r=.78, p<.001)$ and P3 peak amplitude $(r=-.79, p<.001)$.

$>$ In the rhythmic experiment : The same brain component (IC-D) did not significantly $(p>.20)$ disentangle CONTINUE vs. STOP conditions (panel $D)$.

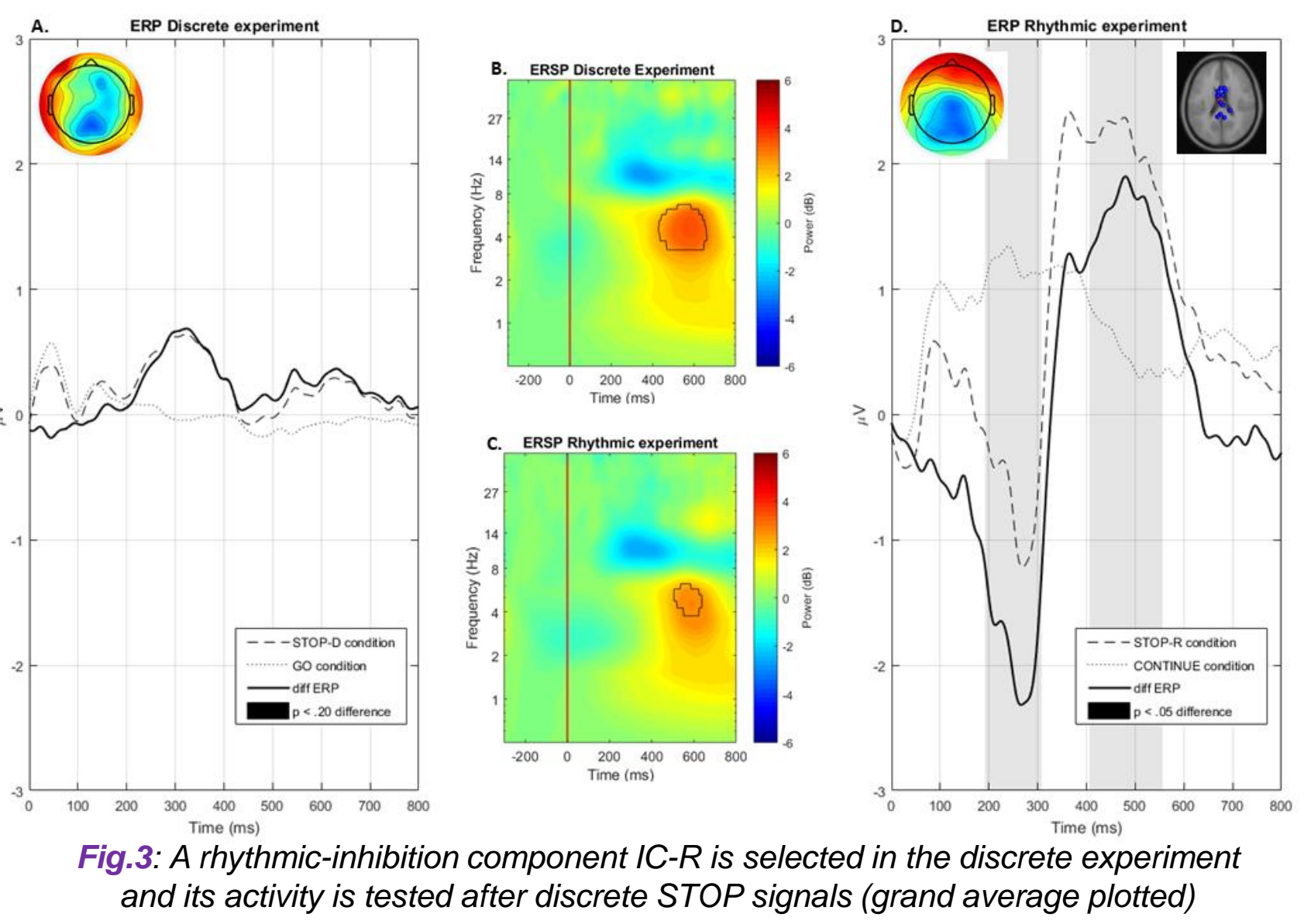

\section{Source estimation of the brain inhibitory components}

Discrete and rhythmic inhibitory component source estimations were compared (Fig.4).

Increased activity was observed in precentral gyri and posterior parts of the cingulate cortex for discrete-action cancelling (vellow), whereas a higher activity was found in more frontal gyri and anterior parts of the cingulate cortex for rhythmic-action stopping (blue).

Fig.4: Source localization analysis: IC-D versus IC- $\boldsymbol{R}$ ( $t$-values, $p<.01$ corrected for multiple comparisons) (grand average plotted)

$>$ The brain component that best represents discrete-action inhibition is not involved in continuous-action inhibition.

$>$ Reversely, the brain component that best represent continuous-action inhibition is not involved in discrete-action stopping.

$>$ This dissociation is supported by differences in the neural implementation of inhibition, especially in the cingulate cortex.

$>$ The brain inhibitory network may engage distinct activity as a function of the action type to revise. 